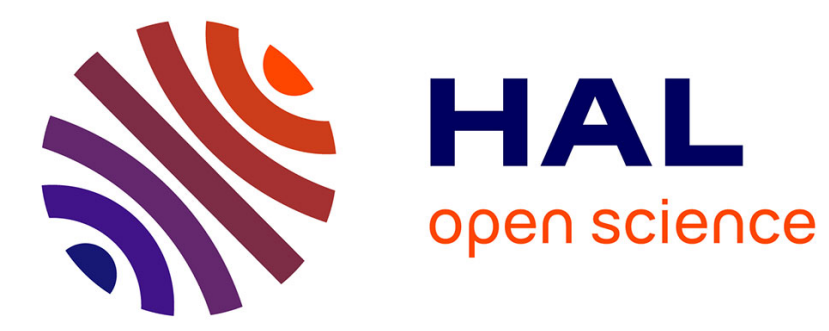

\title{
Le patrimoine culturel immatériel à Tonga et ses médiations touristiques
}

Aurélie Condevaux

\section{To cite this version:}

Aurélie Condevaux. Le patrimoine culturel immatériel à Tonga et ses médiations touristiques. Culture et Musées, 2014, 23, pp.43-65. hal-01054472

HAL Id: hal-01054472

https://hal.science/hal-01054472

Submitted on 7 Jul 2021

HAL is a multi-disciplinary open access archive for the deposit and dissemination of scientific research documents, whether they are published or not. The documents may come from teaching and research institutions in France or abroad, or from public or private research centers.
L'archive ouverte pluridisciplinaire HAL, est destinée au dépôt et à la diffusion de documents scientifiques de niveau recherche, publiés ou non, émanant des établissements d'enseignement et de recherche français ou étrangers, des laboratoires publics ou privés. 


\section{Culture \& Musées}

Muséologie et recherches sur la culture

$23 \mid 2014$

Tourisme et médiations des patrimoines

\section{Le patrimoine culturel immatériel à Tonga et ses médiations touristiques}

Intangible Cultural Heritage in Tonga and tourist mediations

El patrimonio cultural inmaterial en Tonga y sus mediaciones turísticas

Aurélie Condevaux

p. $43-65$

https://doi.org/10.4000/culturemusees.1344

\section{Résumés}

Français English Español

L'apparition de la notion de patrimoine culturel immatériel (PCI) est parfois décrite comme participant à l'émergence d'un " nouveau régime de patrimonialité ». À partir de l'exemple du lakalaka tongien, inscrit sur la liste représentative du PCI de (Unesco, il s'agit de s'interroger sur le renouvellement apporté par la convention pour la sauvegarde du patrimoine culturel immatériel (2003) en ce qui concerne les valeurs qui fondaient jusque-là le champ patrimonial, notamment «l'authenticité » et l'idée de transmission à l'identique - et la place du tourisme dans les politiques patrimoniales.

It has been proposed that the notion of intangible cultural heritage (ICH) may contribute to the emergence of a new "heritage regime". Using the example of the Tongan lakalaka, which has been inscribed on the representative list of ICH by Unesco, this paper aims to understand whether the convention for the safeguarding of intangible cultural heritage (2003) brings a real renewal of the founding values of heritage - such as "authenticity" and the idea that cultural practices should be transmitted in a fixed form - and to address the role of tourism in heritage policies.

El surgimiento de la idea de patrimonio cultural inmaterial (PCI) es a veces descrito como partícipe de la emergencia de un "nuevo régimen de patrimonialidad". Partiendo del ejemplo del lakalaka tongano, inscrito sobre la lista representativa del PCI de la Unesco, se trata de interrogarse sobre la renovación aportada por la convención para la conservación del patrimonio cultural inmaterial (2003) en lo que concierne los valores que fundan hasta aquí el campo patrimonial, principalmente 
"la autenticidad" y la idea de idéntica transmisión - y el rol del turismo en las políticas patrimoniales.

\section{Entrées d'index}

Mots-clés : patrimoine culturel immatériel, tourisme, authenticité, Tonga, lakalaka

Keywords: intangible cultural heritage, tourism, authenticity, tonga, lakalaka

Palabras clave: patrimonio cultural inmaterial, turismo, autenticidad, Tonga, lakalaka

Notes de la rédaction

Manuscrit reçu le : 31 mars 2013

Version révisée après expertise, reçue le : 4 novembre 2013

Article accepté pour publication le : 17 décembre 2013

\section{Texte intégral}

Je remercie les personnes ayant accepté de participer à cette enquête à Tonga, ainsi que le ministère de l'Éducation pour m'avoir autorisé à mener celle-ci. Merci à $\mathrm{A}$. et $\mathrm{L}$. Vı et à toute la famille Koloamatangi pour leur hospitalité et soutien indéfectibles. Une partie de cette enquête a été conduite grâce au soutien financier du Labex CAP (Création, arts et patrimoines). Je remercie également le service des archives de l'Unesco à Paris pour m'avoir permis d'accéder aux dossiers cités.

1 La mise en tourisme des éléments du patrimoine culturel immatériel (PCI) tel qu'il a été défini par l'Unesco peut soulever des questions sensiblement différentes de la mise en tourisme, par exemple, des éléments des patrimoines bâtis et monumentaux.

La différence ne vient pas d'une opposition de nature, trop schématique, entre le matériel et l'immatériel, mais plutôt des évolutions de la définition du patrimoine que l'émergence du concept d'immatériel traduit. En effet, comme l'ont souligné de nombreux auteurs, la convention de l'Unesco pour la sauvegarde du patrimoine culturel immatériel adoptée le 17 octobre 2003 par la conférence générale de l'Unesco et entrée en vigueur en 2006 - résulte de la lente intégration des perspectives anthropologiques sur la culture et le patrimoine, conduisant à une vision dynamique de ceux-ci (Bortolotto, $2011: 22-23 ; 28$ ). La convention reconnaît ainsi la possibilité que les œuvres du PCI soient sans cesse recréées par les communautés qui les ont produites. On serait donc passé d'une conception du patrimoine basée sur un discours de l'authentique à une conception du patrimoine comme dynamique, fondée sur l'idée que les pratiques ne doivent pas faire l'objet d'une transmission figée, mais peuvent être adaptées par les générations futures. Pour décrire cette évolution, certains proposent de parler d'un nouveau régime de patrimonialité (Turgeon, 2011) qui valoriserait « la transformation des pratiques culturelles, la performance de la personne et l'expérience sensible de la culture » (ibid. : 390).

3 On peut se demander dès lors comment - dans ce nouveau régime de patrimonialité qui affirme notamment reléguer au passé les questions ayant trait à l'authenticité - les recréations engendrées par la mise en tourisme et le déploiement de dispositifs de médiation sont considérées par les acteurs en charge de l'application des politiques patrimoniales, mais aussi par les membres des communautés eux-mêmes. Cette question naît du constat qu'il existe une contradiction, notée par de nombreux auteurs, dans la politique patrimoniale de l'Unesco, entre le projet patrimonial - fondé sur l'idée qu'il faut sauvegarder les patrimoines, en les préservant notamment des menaces de la marchandisation - et le projet développementaliste - basé sur l’idée que le patrimoine peut servir de ressource dans les politiques de développement (Andrieu, 2010 : 424-426 ; Ciarcia, $2006:$ 66). Alors que le tourisme est bien souvent brandi comme une menace justifiant la nécessité de protéger les pratiques à travers une politique de 
patrimonialisation, il n'en demeure pas moins que la classification sur l'une ou l'autre des listes du patrimoine de l'Unesco équivaut souvent à une labellisation, qui a pour effet une production de valeurs, puisqu'elle serait l'expression de la reconnaissance du caractère traditionnel ou authentique de la pratique. Cette marque de qualité est par conséquent généralement utilisée comme telle dans le marketing touristique (Hafstein, 2009 : 106 ; Hertz \& Chappaz-Wirthner, 2012 : 4). Le paradoxe n'est pas des moindres : alors même que les politiques patrimoniales de l'Unesco sont pensées pour préserver les pratiques des affres de la mondialisation et de la marchandisation, une reconnaissance par l'Unesco entraîne presque automatiquement des formes de marchandisation et d'évaluation économique (Kirshenblatt-Gimblett, 2006 : 195). Cela va même au-delà d'une simple conséquence, puisque le développement touristique est fréquemment un but, bien qu'inavoué, des candidatures à l'Unesco (Hafstein, 2009: 106).

4 On pourrait s'attendre, pour plusieurs raisons, à ce que l'émergence de la notion de patrimoine culturel immatériel et la convention de 2003 lèvent certaines des ambivalences des politiques patrimoniales à l'égard du tourisme. Tout d'abord, comme évoqué précédemment, la définition du PCI tend, en apparence du moins, à s'écarter d'un régime de patrimonialité basé sur l'idée qu'il est possible de distinguer "l'authentique » de «l'inauthentique », le « vrai » du « faux », etc. (Bortolotto, 2007 : 27). Ensuite, comme le souligne Saskia Cousin (2008), dans l'appréhension de la culture qui s'est développée au sein de l'Unesco à partir des années quatre-vingt-dix - en lien étroit avec la définition du patrimoine culturel immatériel -, le tourisme fut peu à peu envisagé comme faisant partie intégrante des processus de revitalisation des cultures. Dans cette perspective, « il ne s'agit en effet pas moins que de considérer le tourisme comme un élément de constitution des identités culturelles post-nationales » (Cousin, $2008: 51$ ). Enfin, la transformation du patrimoine en " ressource » de développement (Andrieu, 2010), et en particulier en ressource touristique, serait d'autant plus susceptible de se produire dans le cas du PCI que les éléments relevant de cette catégorie seraient par leur « nature » même - souvent « spectaculaire »- plus facilement montrables à un public extérieur (Maguet, 2001: 61).

5 Malgré tout, le spectre d'un régime de patrimonialité fondé sur l'authenticité et la stigmatisation du tourisme n'est pas loin. Alors que la définition du PCI laisse entendre que celui-ci, " transmis de génération en génération, est recréé en permanence par les communautés et groupes en fonction de leur milieu, de leur interaction avec la nature et de leur histoire » (Unesco, $2003:$ 2), le préambule de la convention reconnait en même temps que « les processus de mondialisation et de transformation sociale [...] font, tout comme les phénomènes d'intolérance, également peser de graves menaces de dégradation, de disparition et de destruction sur le patrimoine culturel immatériel, en particulier du fait du manque de moyen de sauvegarde de celui-ci » (ibid. : 1). Par cette rhétorique, la convention de 2003 établit une distinction entre des « recréations » acceptables - celles liées aux milieux dans lesquels les communautés vivent et à leur histoire - et des changements non acceptables - ceux engendrés par la mondialisation et les " phénomènes d'intolérance ». La mondialisation se trouve ainsi placée hors de l'histoire - même contemporaine bien sûr - des « communautés ». Le tourisme, même s'il n'est pas mentionné directement dans les extraits de la convention cités ci-dessus, risque de tomber sous l'opprobre de cette déclaration, en tant que phénomène lié à la mondialisation. Il y a donc lieu de se demander si, parmi les « recréations » jugées possibles et acceptables par la définition du PCI, celles liées à la médiation touristique ne resteraient pas indésirables. Les nouvelles manières d'envisager le patrimoine qui ont fait jour au cours des dernières décennies permettent-elles de dépasser les tensions évoquées précédemment entre les politiques de sauvegarde du patrimoine et celles qui visent plutôt sa mise en valeur par le tourisme?

6 Poser ces questions suppose de se départir d'une vision dichotomique qui renverrait dos à dos le processus de patrimonialisation et celui de la mise en tourisme au motif qu'il y aurait d'un côté une culture " intacte » ou " pure », qui devrait faire l'objet de la patrimonialisation, et de l'autre une culture " mise en tourisme », dévoyée parce que 
marchandisée. Au contraire, mise en tourisme et patrimonialisation gagnent à être abordées comme deux processus concomitants qui peuvent, sous certains aspects, s'enrichir mutuellement. En effet, si la «mise en tourisme » ou la « valorisation » du patrimoine nécessitent des stratégies de médiation et d'autres formes d' " adaptations » de la pratique, cette formulation tend à masquer que le " patrimoine » lui-même n'est pas un donné. La position adoptée dans cet article s'écarte du point de vue selon lequel le patrimoine ne serait que l'héritage du passé pour s'inscrire dans la lignée des travaux qui considèrent plutôt celui-ci comme une construction s'effectuant dans le présent (Rautenberg, 2003), à travers des choix façonnés notamment par les rapports de force et les luttes sociales actuelles (Munz, 2012). Le tourisme lui-même peut d'ailleurs être un facteur clef de la construction du patrimoine (Noppen \& Morisset, 2003), l'expression « machine à produire des patrimoines » (Gravari-Barbas, 2009: 76) traduisant utilement cette réalité, même si ce mécanisme, nous le verrons, ne permet peut-être pas d'expliquer la patrimonialisation du lakalaka tongien, qui est l'objet de cet article.

7 Plutôt que de reprendre la sémantique de l'Unesco, la réflexion proposée part de l'hypothèse que l'opposition entre sphère marchande - touristique - et sphère patrimoniale est une construction sociale. À partir de là, l'enjeu de l'étude des médiations touristiques du patrimoine culturel immatériel devient un angle d'approche pertinent pour comprendre si l'émergence de cette notion participe bel et bien à l'avènement d'un nouveau régime de patrimonialité, ou si les continuités sont plus profondes qu'on ne le pense. Le développement touristique est envisagé ici comme un révélateur des conceptions antagonistes de l'identité et du patrimoine, et notamment un révélateur de ce que devient la question de l'authenticité - et les oppositions qui l'accompagnent généralement, comme celles entre le vrai et le faux, le modèle et la copie - dans l'application d'une convention affirmant se départir de celles-ci. À ce titre, nous verrons que, plutôt que d'un unique régime de patrimonialité, il serait sans doute préférable de parler de " régimes patrimoniaux » (Bendix, Eggert \& Peselmann, 2012) afin de mieux saisir ce qui se perd ou se crée - dans l'application concrète d'une convention internationale. Au-delà d'une interrogation sur le régime de patrimonialité dans lequel nous sommes ou serions entrés, il s'agira également de comprendre en quoi la manière dont les limites sont placées entre recréations acceptables et non acceptables peut nous renseigner sur les dynamiques sociales à l'œuvre dans un contexte social donné.

8 Le contexte qui nous intéresse plus particulièrement ici est celui de la société tongienne et les questions évoquées seront examinées à l'aide de l'exemple du lakalaka, forme de poésie chantée et dansée, qui a été déclarée chef-d'œuvre du patrimoine oral et immatériel de l'humanité en 2003 puis inscrite sur la liste représentative du PCI par l'Unesco en 2008. Celui-ci présente un cas d'étude particulièrement fécond pour aborder les questions soulevées ci-dessus dans la mesure où, comme il sera expliqué plus loin, sa patrimonialisation n'a pas été motivée par des perspectives de développement touristique. Les hypothèses avancées reposent sur une étude antérieure du passage des lakalaka des contextes " cérémoniels » aux contextes «touristiques », laquelle utilisait des données recueillies au cours d'enquêtes de terrain menées à Tonga en 2008 et 2009 - sept mois au total. Les méthodes employées reposaient notamment sur l'observation participante, en particulier à travers la participation à un lakalaka en 2008, lors du couronnement du roi George Tupou V - aujourd'hui décédé. Ces sessions d'enquête de terrain ont également été l'occasion d'assister à de nombreux lakalaka dans des contextes touristiques ou lors de compétitions ou de fêtes religieuses, ainsi que de mener des entretiens ou conversations avec des punake - experts en composition musicale et poétique et en chorégraphie. Cet article propose d'aller plus loin en examinant les enjeux de la " mise en tourisme » du lakalaka du point de vue de la patrimonialisation de la pratique à l'aide de nouvelles données recueillies au cours de deux séjours à Tonga, en 2012 et 2013, auprès des acteurs patrimoniaux. Enfin, des documents d'archive de l'Unesco, consultés à Paris, et la littérature qui traite du PCI, abondante malgré le caractère récent de la convention, sont également utilisés. Une première partie concerne le contexte tongien. Dans un second 
temps nous verrons, après une description de la pratique dans ses différents contextes d'apparition, quelles sont les stratégies de mise en scène utilisées pour montrer les lakalaka à un public extérieur. Enfin, nous verrons comment ces " adaptations » sont interprétées et commentées par les différents acteurs impliqués dans la pratique du lakalaka et/ou dans sa patrimonialisation.

\section{Le lakalaka tongien et sa patrimonialisation}

\section{Le contexte tongien}

Tonga est un archipel d'un peu plus de cent-soixante-dix îles situé à environ $2100 \mathrm{~km}$ au nord-est de la Nouvelle-Zélande. Il fait partie d'une aire géographique généralement qualifiée de « polynésienne » suite à la cartographie élaborée par les premiers navigateurs européens, sur des critères à la fois « raciaux » et culturels (Tcherkézoff, 2003). L'archipel tongien, jamais colonisé - mais "protégé » par la Grande-Bretagne durant soixante-dix ans -, a été unifié par le roi Tâufa'āhau George Tupou I au XIXe siècle. Ses descendants, aujourd'hui à la tête de l'État, se revendiquent comme les héritiers des lignées de chefs les plus prestigieuses, y compris celle du Tu'i Tonga - chef suprême -, dont l'histoire orale situe le règne du premier représentant au Xe siècle - bien que, selon certains, les huit premiers noms de la lignée des Tu’i Tonga soient mythiques (Kirch \& Wood, in DouaireMarsaudon, 1998 : 20). Pour ces différentes raisons, Tonga est généralement décrit comme le dernier royaume polynésien du Pacifique, dans une perspective qui met l'accent sur la continuité des traditions. Il est vrai que l'opposition entre les hoüeiki et les tüa ou kakai, des termes généralement traduits par «nobles » et « gens du commun », reste l'un des principes structurants les plus forts du système social tongien - à côté d'autres critères comme celui de l'âge. Les grandes fêtes ( $k a \bar{t} t o a n g a)$ qui marquent les événements de la vie de la famille royale - anniversaires, mariages, et bien sûr couronnement - continuent de rythmer la vie du royaume, et en particulier celle de Tongatapu, l'île principale de l'archipel. C'est là que vivent la plupart des familles nobles, lorsque celles-ci n'ont pas choisi de vivre en Australie, en Nouvelle-Zélande ou aux États-Unis.

En effet, comme le souligne Niko Besnier (2011), la société tongienne ne peut être définie comme étant uniquement constituée de la population résidant dans cet archipel du Pacifique sud. On estime à 100000 à 250000 le nombre de Tongiens vivant en Australie, aux États-Unis et en Nouvelle-Zélande (ibid. : 42), alors que la population de Tongiens vivant à Tonga est d'à peine plus de 103000 selon les dernières estimations.

La société tongienne expérimente des changements importants, qui trouvent leurs sources aussi bien dans ces diasporas qu'à Tonga - changements qui, ces dernières années, se sont notamment traduits sur le plan politique. Jusqu'en 2010, la majorité des députés était constituée de nobles élus par des nobles ou de représentants nommés directement par le roi. Le mouvement démocratique, fondé dans les années quatre-vingt, notamment par des roturiers ayant suivi des études supérieures à l'étranger, et fortement encouragé par les puissances étrangères (Besnier, 2011 : 3), a peu à peu affaibli le pouvoir royal - non sans quelques heurts violents comme les émeutes qui eurent lieu dans la capitale tongienne, Nuku'alofa, le 16 novembre 2006. Ce soulèvement fut suivi d'une accélération de la réforme politique, marquée notamment par un abandon partiel des pouvoirs par le monarque et une transformation du mode d'élection du Parlement : en 2010, pour la première fois dans l'histoire du pays, la majorité du Parlement - dix-sept sièges - était élue par voie de suffrage universel, alors que neuf sièges revenaient aux nobles. 
Si les sources du changement social sont multiples, le tourisme ne semble pas faire partie des principaux facteurs. Dans les années cinquante et soixante, quelques milliers de visiteurs seulement - moins de 2000 en 1958 par exemple - se rendaient chaque année dans le petit archipel (Teilhet-Fisk, 1991 : 43). Aujourd'hui, le tourisme international est encore relativement discret, surtout si on le compare à quelques destinations voisines du Pacifique comme les îles Cook, Hawai ou la Polynésie française. En revanche, les Tongiens qui habitent dans les diasporas du pourtour du Pacifique se rendent fréquemment dans leur pays d'origine, que ce soit pour un événement particulier ou simplement pour visiter leurs familles.

Contrairement à ce qui a pu être observé dans d'autres régions du monde, la candidature déposée auprès de l'Unesco pour le lakalaka ne semble pas avoir été motivée par des perspectives de développement touristique. En effet, de manière assez surprenante, l'inscription de cette pratique sur la liste représentative du PCI n'a pas été suivie d'une valorisation spécifique dans l'argumentaire touristique, contrairement à ce que l'on peut observer ailleurs (Noyes, 2011). En revanche, d'autres enjeux peuvent expliquer sans nul doute la patrimonialisation du lakalaka, comme les changements intervenus dans le système politique au cours des dernières décennies et les défis rencontrés par le pouvoir en place. En effet, la pratique est en elle-même très étroitement liée au système hiérarchique.

\section{Le lakalaka aujourd'hui}

Le lakalaka est l'une des dix catégories de performances dansées et chantées reconnues d'un point de vue émique à Tonga. Comme la plupart des performances pratiquées dans cet archipel du Pacifique et dans les diasporas tongiennes, le lakalaka mêle une poésie chantée à une gestuelle dansée, les gestes venant illustrer les paroles. La poésie ainsi récitée délivre généralement un commentaire sur l'événement en cours - le plus souvent une cérémonie royale de type mariage, anniversaire ou couronnement. Aujourd'hui, les lakalaka sont pratiqués dans des contextes variés, dont certains entretiennent peu de rapport avec la structure hiérarchique de la société tongienne, comme des fêtes religieuses ou des scènes touristiques. Les danseurs, qui sont aussi des chanteurs, sont alignés face au public, hommes d'un côté, femmes de l'autre, bien que les rangs puissent occasionnellement se croiser. Danseurs et danseuses, qui chantent tout en dansant, sont aussi souvent accompagnés par un chœur de chanteurs - appelé langitu'a - placé à l'arrière du groupe.

15 Lorsqu'elles sont produites dans des contextes cérémoniels, l'un des traits caractéristiques essentiels de ces manifestations esthétiques est le rôle qu'elles jouent dans l'expression et la reproduction des structures hiérarchiques de la société (Kaeppler, 1993). Les participants à un lakalaka ne font pas l'objet d'une sélection sur des critères de rang, d'âge ou de sexe : tous, dans le village, le quartier ou l'église concernée, peuvent participer. En revanche, ces critères organisent la disposition des danseurs les uns par rapport aux autres. Certaines places sont ainsi réservées aux personnes de plus haut rang : ce sont en particulier les positions les plus centrales sur le premier rang, à savoir les positions 1, 2, 5, 7 et 10, du côté des hommes comme du côté des femmes, ainsi que la dernière sur le rang, en numérotant « 1 » les positions les plus centrales. Les deux personnes, homme et femme, qui occupent les positions les plus au centre sont appelées vàhenga (Condevaux, 2011).

16 Comme le dit Adrienne Kaeppler, durant la préparation d'un lakalaka, " un individu est agréablement "enculturé" aux valeurs consacrées de cette société, c'est-à-dire qu'il apprend les rôles sociaux et l'importance relative des chefs, matāpule - porte-parole de chef - et tu'a - roturier -, les statuts qui sont assignés à la naissance, et la mobilité rendue possible par la réussite ${ }^{2} »(1993: 58)$. La préparation, qui commence des semaines, voire des mois à l'avance dans un hall du village, rassemble d'abord peu de personnes, le 
nombre des participants augmentant au fur et à mesure des répétitions. L’incertitude règne au début quant à savoir qui se tiendra aux différentes positions de " prestige » mentionnées précédemment, le suspens pouvant durer jusqu'au dernier moment. Ces positions sont en effet l'objet de convoitises : ceux qui veulent les occuper doivent pouvoir rappeler leurs généalogies et leurs liens de filiation avec des figures historiques ou vivantes de la noblesse tongienne.

17 Pour les personnes qui occuperont les positions de vāhenga - positions les plus centrales -, il arrive que le punake se déplace au domicile de la personne en question durant la période de répétition, eu égard au rang de celle-ci, afin de lui apprendre les premiers rudiments de la chorégraphie. Néanmoins, ces nobilités peuvent venir à leur tour participer à des répétitions communes. C'est alors l'occasion de leur offrir des rafraîchissements spéciaux, un accueil enthousiaste ou même des divertissements au moment des pauses - autant de manières de marquer leur statut.

Les paroles de la composition sont un autre élément essentiel de la célébration des lignées royales (Kaeppler, 1993 : 96). Les lakalaka débutent par un fakatapu - strophe d'ouverture - qui permet de rendre hommage aux nobles présents dans l'audience. En voici ci-dessous un exemple : dans un lakalaka qu'elle a composé en 1928 pour le départ de son fils aîné en Australie, la reine Sâlote, qui était une punake de renom, fît un lakalaka connu sous le nom de "Takafalu »- terme métaphorique pour désigner le dos du monarque. Ce lakalaka commence par les lignes qui suivent (paroles et traduction anglaise : Wood-Ellem, 2004: 260):

\begin{tabular}{|c|c|}
\hline Ke fakatulou mo e takafalu & Rendons hommage au Dos du Monarque \\
\hline Mo e 'otu laine toputapu & Et à la chaîne sacrée des lignées \\
\hline Ne fetaulaki 'o tapa tolu & Qui se rencontrèrent et formèrent le triangle \\
\hline Holo pē nofo he lau 'otu & Laissez-vous porter dans la hiérarchie \\
\hline Ne kamata 'ia 'Aho'eitu & Qui commença avec Aho'eitu \\
\hline Afe he tuliki Fonuamotu & Tourna à l'angle de Fonuamotu \\
\hline Tu'u mo e tapa 'i 'Āhau & Tint bon à la faction de 'Ahau \\
\hline Piliote 'i Pangai e fa'u & Et finit dans la structure de Pangai \\
\hline
\end{tabular}

La reine, en exprimant de la déférence pour le « dos du monarque », souligne ainsi que son fils est l'héritier des principales lignées de chef - la " chaîne sacrée des lignées » - qui ont dominé l'histoire de Tonga. 'Aho'eitu - cinquième vers - est en effet le nom du premier Tu’i Tonga qui aurait régné sur l'archipel - bien que, comme évoqué précédemment, il soit possiblement mythique. Le sixième vers renvoie quant à lui à la lignée des Tu’i Ha'atakalaua. Celle-ci émergea au XVIe siècle, pour des raisons encore discutées : alors que certains avancent que le Tu’i Tonga délégua volontairement une partie de ses pouvoirs, d'autres supposent au contraire qu'ils lui furent usurpés (DouaireMarsaudon, 1998 : 21-22). Toujours est-il que les Tu’i Ha'atakalaua eurent longtemps le contrôle des pouvoirs séculiers et militaires, avant de perdre à leur tour une part de leur influence face à la montée d'une troisième lignée, celle des Tul Kanokupolu. D’après Melenaite Taumoefolau (2004 : 115), "Fonuamotu » désigne un lieu dans Mu'a l'ancienne capitale tongienne où résidèrent aussi bien les Tul Tonga que les Tul Ha'atakalaua - très étroitement lié à cette dernière lignée. Pour cette raison, ce terme est souvent utilisé par la reine pour désigner les héritiers des Tul Ha'atakalaua, dans une utilisation classique, dans la poésie tongienne, de figures métaphoriques pour faire référence de manière indirecte aux personnes de haut rang. 'Ahau est un village près de Kanokupolu, sur la côte ouest de l'île principale, Tongatapu. D'après une informatrice, la référence à ce village renverrait ici à la lignée des Tu’i Kanokupolu. Ces différents éléments indiquent tous que, lors des grands kātoanga qui concernent la famille royale, les 
lakalaka sont donc à la fois un moyen de socialiser les individus qui y participent au système hiérarchique tongien dans toute sa complexité, et d'exprimer, à la vue des spectateurs, la supériorité des hou'eiki, et en particulier des membres de la famille royale, vis-à-vis des tu'a ou kakai.

Comme évoqué précédemment, les lakalaka sont aujourd'hui parfois mis en scène dans des contextes touristiques, mais aussi lors de compétitions ou de fêtes religieuses. Ces contextes de performances, qui ne sont pas nécessairement nouveaux, ne semblent pas directement découler de la reconnaissance de la pratique par TUnesco. Arrêtons-nous quelques instants sur cette classification et ses effets. Suite à la reconnaissance du lakalaka comme chef-d'œuvre du patrimoine oral et immatériel de l'humanité en 2003, le gouvernement tongien se vit accorder un budget de 120 ooo US\$ pour réaliser son plan de sauvegarde. Celui-ci, réalisé par le Ministry of Education, Women Affairs and Culture MEWAC, ministères qui a depuis été divisé en plusieurs départements ou autres ministères - et le Tonga Tradition's Committee, avait pour principaux objectifs d'effectuer une recherche sur les compositions existantes de lakalaka et sur les personnes impliquées dans sa pratique - travail principalement effectué entre 2005 et 2008 -, de publier les résultats de ces recherches et d'organiser des événements permettant de revitaliser la pratique - festivals -, notamment auprès des jeunes.

En revanche, d'une manière assez surprenante, aucune action ne fut envisagée pour valoriser la reconnaissance de l'Unesco auprès des touristes : pas plus en 2013 qu'en 2008, le lakalaka n'est mentionné dans les brochures réalisées par le Tourism Visitors Bureau (TVB) ou le guide Jason Travel Media, qui est l'une des principales brochures touristiques distribuée à Tonga. Les principaux lieux de promotion touristique à Nuku'alofa (le TVB et Friends Café) n'en parlent pas. Lorsque le lakalaka est mis en scène pour des touristes, cela ne résulte pas d'une volonté politique et aucun maitre de cérémonie - personne chargée de présenter les éléments culturels mis en scène - des performances touristiques auxquelles j'ai assisté ne mentionnait, lors de son introduction du lakalaka, la distinction obtenue auprès de l'Unesco. Si la valeur patrimoniale attribuée au lakalaka via cette labellisation n'est donc pas mise en avant dans le marketing ou sur les scènes touristiques, les lakalaka n'en sont pas moins présents dans ces contextes.

Ces présentations interviennent dans le cadre de performances touristiques ${ }^{3}$ constituées à la fois d'une démonstration de musiques et de danses et d'un repas cuit au four semienterré, appelé 'umu à Tonga. Parmi les danses présentées, la plupart s'effectuent en petits groupes voire en solo - par exemple les danses appelées mako et tau'olunga. Le lakalaka en revanche n'est pas un faiva - performance dansée et chantée - très prisé dans ces contextes, sans doute en raison des contraintes que représente son adaptation au contexte touristique $-c f$ ci-après. Les dispositifs utilisés pour le présenter au public sont de deux ordres : 1. des adaptations structurelles à l'espace scénique ; 2. des discours explicatifs délivrés par les maîtres de cérémonie. Deux aspects que je vais détailler à présent.

\section{Les dispositifs de médiation touristique du lakalaka}

\section{Adaptations scéniques}

Du point de vue des éléments strictement structurels de la performance, on note des différences relativement importantes entre le contexte des grandes cérémonies royales mentionnées précédemment et celui des scènes touristiques. D'abord, le nombre de danseurs et danseuses est souvent fortement restreint : de plusieurs centaines, voire un millier, dans le cadre d'un contexte cérémoniel, le nombre de danseurs et danseuses passe 
à une quinzaine tout au plus dans les contextes touristiques. Cela s'explique bien sûr par l'impossibilité de rassembler un si grand nombre de pratiquants pour des représentations régulières, qui ont lieu une ou plusieurs fois par semaine - d'autant plus que ces derniers sont rémunérés, même faiblement. La performance se déroule en outre sur un espace relativement limité, celui d'une scène surélevée par rapport au niveau du sol, alors que le groupe de danseurs se tient généralement sur un espace dégagé, au même niveau que le public - voire plus bas que celui-ci - dans le cadre d'un contexte « cérémoniel ». Malgré ce nombre restreint de participants, danseurs et danseuses sont généralement répartis en deux groupes, d'un côté les hommes et de l'autre les femmes, tout comme c'est le cas lors des cérémonies.

Pour ce qui est des mouvements exécutés, on note une assez grande similarité entre les contextes touristiques et cérémoniels : le pas " de base »-qui consiste à effectuer un déplacement latéral alternativement à gauche puis à droite -, la présence de mouvements de bras et de mains qui sont souvent - mais pas toujours - reproduits de manière symétrique d'un côté puis de l'autre, et la présence du fü - un geste qui consiste à frapper les paumes des mains l'une contre l'autre -, en particulier en fin de chaque couplet.

La durée de la performance est généralement réduite. Alors que, dans les contextes cérémoniels, les lakalaka peuvent durer entre dix et vingt minutes - trente minutes d'après certains, même si cela semble plus rare -, sur les scènes touristiques au contraire ils sont réduits à trois à cinq minutes. Cela est rendu possible en ne répétant pas chaque couplet deux fois - contrairement à ce qui est fait dans le cadre d'un kātoanga - et/ou en n'interprétant pas tous les couplets de la composition. Sur les scènes touristiques, le chœur de chanteurs est d'ailleurs souvent réduit à quelques personnes, ce qui ne permet pas toujours de représenter toute la variation des tonalités de chant que l'on trouve habituellement dans la polyphonie des lakalaka. Ces différentes adaptations ne sont pas propres au contexte touristique puisqu'elles se retrouvent dans d'autres occasions compétitions, fêtes religieuses, etc.

Outre ces changements scéniques, la mise en scène touristique induit des changements dans les relations mises en ouvre pour " produire » le lakalaka. Comme le souligne Jehanne H. Teilhet-Fisk (1991), les études sur la mise en tourisme des pratiques esthétiques se sont souvent focalisées sur l'impact du tourisme sur les aspects purement formels et esthétiques de l'objet, au détriment des changements plus subtils que l'« acculturation » - c'est le terme qu'elle utilise - peut apporter dans les significations socioculturelles et symboliques de celui-ci. En ce qui concerne le lakalaka, si l'on souhaite comprendre les enjeux du passage du contexte cérémoniel à celui des scènes touristiques, des compétitions, ou d'événements religieux, on ne peut se contenter d'analyser les changements formels. Il faut aussi prendre en compte ceux qui interviennent dans les relations mises en place pour produire cette pratique.

27 Dans les performances touristiques tongiennes, les danseurs et danseuses mobilisés pour les lakalaka, - ceux-là même qui exécutent l'ensemble des autres danses -, sont de jeunes gens généralement non mariés, roturiers, et qui ne représentent donc pas la diversité de la société tongienne et des positions hiérarchiques existantes. Les hou'eiki «nobles » - étant absents de ces groupes, le positionnement des danseurs les uns vis-à-vis des autres n'est pas déterminé en fonction de critères hiérarchiques et les répétitions ne sont pas non plus l'occasion de rappeler les généalogies des familles nobles. Si l'expression ou la reproduction des hiérarchies de rang ne sont plus au cœur des lakalaka pratiqués dans ces différents contextes, cela ne signifie pas que ceux-ci sont dépourvus de signification sociale : ils en revêtent en effet de nouvelles, qui leur sont conférées par les personnes qui les pratiquent, et que l'on peut trouver dans les discours entourant la performance (Condevaux, 2011). Lors des performances touristiques par exemple, les lakalaka deviennent un moyen de souhaiter la bienvenue aux visiteurs étrangers, à travers des textes poétiques composés spécifiquement pour cet événement. 


\section{Dispositifs de médiation discursive}

Les dispositifs de médiation déployés lors de la mise en scène des lakalaka dans les contextes touristiques incluent des éléments discursifs qui, tout en donnant des éléments d'information que l'on pourrait qualifier de "factuels ", offrent également une " mise en signification » de la pratique qui n'est pas neutre. Dans certaines performances, les lakalaka sont présentés de manière à « rappeler » la fonction qu'ils ont dans un contexte cérémoniel, en soulignant leur rôle dans les grandes cérémonies royales (Condevaux, 2011 : 284). Cela permet de donner un « contexte » de compréhension pour les touristes qui n'ont jamais entendu parler de lakalaka auparavant. Parfois, ce rappel en anglais peut être " doublé » d'un hommage rendu aux lignées royales dans les paroles. Bien sûr, ce rappel oral ne remplace pas le rôle que joue la préparation d'un lakalaka dans la reconnaissance effective du statut des hou'eiki - nobles ou aristocrates - et dans l'apprentissage du rang de chacun.

D'autres manières d'introduire les lakalaka ne font en revanche pas référence à leur rôle dans les fêtes royales. Après avoir insisté sur le fait que la tradition est plus préservée à Tonga que dans d'autres archipels du Pacifique - les exemples pris sont Fidji, Samoa et la Polynésie française -, un maître de cérémonie qui introduit un lakalaka souligne par exemple :

Mesdames et Messieurs, la prochaine danse est un lakalaka. Les lakalaka étaient
exécutés pour les anniversaires du roi, ou celui du prince héritier, ou pour un
couronnement. Il pouvait y avoir des milliers de danseurs. Voyez-vous, pour les
jeunes Tongiens, je veux qu'ils sachent que nous sommes le vrai Pacifique. Je ne dis
pas que nous devrions crâner. Ce que je veux dire, c'est que l'on devrait rester
comme ça, ok les gars? Gardez ça en tête. Vous voyez les femmes, couvertes de haut
en bas. Les Tahitiennes, les Fidjiennes, les Samoanes racontent une histoire avec
leurs hanches. Pas à Tonga. (Discours de présentation d'une performance
touristique, Tongatapu, juin 2012.)

Dans ce discours, le rôle de la famille royale est relégué au passé, chose étonnante dans la mesure où le système politique demeure monarchique et que, lors des cérémonies royales les plus récentes, des lakalaka rassemblant plusieurs centaines de danseurs furent exécutés : ce fut par exemple le cas lors du couronnement du roi George Tupou V en 2008, de son anniversaire en 2009, ou encore du mariage princier qui eut lieu en juillet 2012 pour célébrer l'union du nouveau prince héritier ('Ulukalaka) et de sa fiancée (Sinaitakala). Ici, le maître de cérémonie exprime des craintes quant à la préservation des traditions et interpelle les jeunes Tongiens pour souligner l'importance qu'il y a à transmettre et pérenniser ces dernières. Il met en avant le fait que l'une des caractéristiques de l'identité tongienne - en comparaison à d'autres archipels du Pacifique - reposerait sur "l'authenticité », au sens de " vérité » : " le vrai Pacifique », de cette culture, qui représenterait donc l'« essence » du Pacifique, incarnée par des femmes pudiques et réservées, à l'opposé des images souvent mises en avant dans le marketing touristique de jeunes femmes fortement dénudées.

Ce discours de présentation montre que les changements structurels imposés au lakalaka lors de son passage à la scène touristique s'inscrivent dans des dynamiques identitaires complexes : les lakalaka adaptés aux scènes touristiques présentent certes un contraste important avec ceux des contextes cérémoniels dans la mesure où ils ne permettent pratiquement plus d'exprimer ni de reproduire les hiérarchies sociales. Mais cela s'accompagne de nouvelles manières de définir l'identité tongienne. Les dispositifs de représentation et de communication mis en place pour faire figurer les lakalaka sur les scènes touristiques ne doivent donc pas nous amener à questionner "l'authenticité » de ceux-ci : dans chacun des contextes mentionnés, les lakalaka revêtent une signification particulière et servent de support à une définition spécifique de l'identité tongienne. S’il ne s'agit pas d'émettre un jugement sur l'« authenticité » de ces stratégies de médiation 
touristique, il est néanmoins utile d'examiner comment celles-ci sont reçues et perçues par les acteurs eux-mêmes.

\section{Points de vue sur les « récréations » du lakalaka}

Les attitudes des acteurs face aux adaptations des lakalaka aux contextes touristiques sont très diverses. Plusieurs punake interviewés, qui travaillaient ou non pour des spectacles touristiques, insistaient sur le fait qu'il n'existait à leurs yeux pas de différence entre les lakalaka faits pour le tourisme et les autres, si ce n'est le nombre de danseurs présents. Tout en minimisant ainsi les changements imposés à la forme des lakalaka, ils reconnaissaient la possibilité que leur fonction, en changeant de contexte, évolue, cette évolution étant plus constatée que déplorée, comme il apparaît dans les propos de ce punake responsable de la préparation d'un lakalaka pour le couronnement de 2008 :

T. : À l'origine, [le lakalaka] était exécuté pour la famille royale, mais cela ne signifie pas que c'est uniquement destiné au roi et à sa famille. Ça peut être fait n'importe où, pour n'importe qui. C'est quelque chose que vous verrez toujours. Oui on peut le faire dans un floorshow pour touristes. (Entretien réalisé à Nuku'alofa en août 2008.)

Ce type de point de vue est minoritaire, la grande majorité des punake interrogés avançant qu'à leurs yeux, les lakalaka faits pour les touristes ne méritent pas grande considération. Par exemple, une punake qui avait pourtant elle-même composé un lakalaka pour un floorshow n'en regardait pas moins les représentations touristiques avec peu de bienveillance. Cette punake insistait sur le fait que les scènes touristiques n'étaient pas des contextes "normaux » d'exécution, mais qu'elle-même avait été poussée à faire cette composition pour deux raisons : d'une part parce qu'elle était apparentée au gérant du lieu du spectacle, qui lui en avait fait la requête, et d'autre part parce qu'elle préférait composer un lakalaka « correct » que laisser d'autres le faire au risque de les voir porter atteinte à la «tradition ». Sa composition était donc pour elle comme un moyen de " parer au pire » :
Je n'approuve pas tellement les floorshows vous savez. Parce que ça dépend ce qu'ils enseignent, ils exécutent des danses d'autres îles du Pacifique. Parfois ils font de nouvelles créations. Ce ne sont pas de vraies danses tongiennes traditionnelles. Donc [...] je me suis dit que ça serait bien que je compose pour ce floorshow, sinon ils allaient commencer à faire toutes ces nouvelles compositions, vous savez. C'est pourquoi je devais composer, je devais faire un court lakalaka. (Entretien réalisé à Nuku'alofa en juin 2012.)

Le point de vue plutôt négatif de cette punake, qui a en outre travaillé, selon ses dires, à la rédaction de plusieurs rapports destinés à l'Unesco - sur les lakalaka exécutés lors du couronnement de 2008 -, semble être partagé par les personnes impliquées dans les politiques patrimoniales tongiennes.

Les témoignages des individus ayant participé au travail de documentation et/ou à l'organisation d'événements autour du lakalaka dans le cadre de la mise en application du plan d'action ont été recueillis en 2012 et 2013. Ces personnes travaillaient soit au ministère des Affaires intérieures - dont dépend désormais la Culture -, au ministère de l'Éducation, à la Commission nationale de l'Unesco à Tonga ou au Tradition's Committee - un des organismes impliqués dans la mise en œuvre du plan d'action. L'analyse des entretiens laisse penser qu'à leurs yeux également, les lakalaka pratiqués dans les contextes touristiques sont tout au plus des « aperçus » de la vraie pratique, qui n'ont pas grand intérêt. L'une des personnes impliquée dans le travail de recherche mené sur le lakalaka me disait par exemple, à propos de ceux exécutés sur les scènes touristiques : 
sens, quand tu fais quelque chose d'aussi court. (Entretien réalisé à Nuku'alofa en juin 2013.)

36 Si les termes mêmes « d'authenticité » ou « d'inauthenticité » sont peu utilisés par les acteurs, ils apparaissent néanmoins à plusieurs reprises dans les documents produits par les acteurs en charge de la préservation du patrimoine. Ainsi, dans le Cultural Mapping, Planning and Policy Report, qui est le résultat d'un travail entrepris au sein du MEWAC et financé par l'Union européenne, dont le but était de mener un recensement ou cartographie culturelle et de faire des propositions concernant la politique culturelle, il est souligné que « le floorshow (sic) exécuté pour le divertissement des touristes fait face à des difficultés liées au maintien de la qualité et de l'authenticité, ainsi qu'au développement d'une approche marketing stratégique » (Johannson Fua, Tuita, Kanongata'a \& Fuko, 2011 : 44). La rhétorique de l'authenticité apparaît également dans des documents produits spécifiquement pour l'Unesco. Par exemple, le préambule du Lakalaka Annual Report 2005 affirme que " le lakalaka est la danse tongienne traditionnelle authentique et reconnue » (dossier Unesco CLT/CIH/ITH/111). Le terme, qui semble avoir été proscrit du langage officiel de l’Unesco, n'en est donc pas moins réintroduit.

\section{Conclusion}

37 La convention pour la sauvegarde du patrimoine culturel immatériel, signée en 2003 et entrée en vigueur en 2006, a été pensée comme un moyen de répondre aux critiques dont la convention concernant la protection du patrimoine mondial, culturel et naturel, signée en 1972, avait fait l'objet : on reprochait notamment à cette dernière son caractère eurocentré (Smith \& Akagawa, 2009 : 1-3). La signature de la convention de 2003, motivée par la volonté de rééquilibrer les inégalités Nord-Sud, était fondée sur une remise en question des catégories qui fondaient jusque-là le « champ patrimonial » - le beau, le rare, l'authentique - pour des catégories moins « européanocentrées » (Maguet, 2011 : 47). Il ne s'agissait plus de reproduire des pratiques à l'identique, mais d'assurer les conditions permettant l'adaptation de celles-ci - leurs " recréations » - par les générations futures. De plus, en même temps que la notion de PCI a émergé, celle de « développement durable » a pris de l'importance, devenant un concept clef dans la « constellation de textes » entourant la convention de 2003 (Andrieu, 2010 : 386), textes qui semblent également traduire un nouveau regard sur le tourisme, désormais considéré comme un élément constitutif des dynamiques identitaires contemporaines (Cousin, 2008).

On pouvait penser que ces différentes inflexions apportées par la convention de 2003 s'accompagneraient localement de nouvelles manières d'envisager le tourisme dans les politiques de sauvegarde du patrimoine. Les éléments d'ethnographie présentés ci-dessus montrent que, si «nouveau régime de patrimonialité » (Turgeon, 2011) il y a, celui-ci est adapté en fonction des contextes locaux et ne se traduit pas nécessairement par un changement d'attitude des acteurs patrimoniaux à l'égard du tourisme. Les tensions qui existaient déjà dans la politique du patrimoine mondial entre projets de sauvegarde et projets de valorisation par la mise en tourisme restent d'actualité. Dans le cas du lakalaka, le processus de patrimonialisation se traduit par une stigmatisation des mises en tourisme de la pratique, critiquées notamment pour leur manque "d'authenticité ». Si ce terme apparait peu dans les discours des acteurs recueillis au cours d'entretiens, il apparaît en revanche dans plusieurs documents liés à la politique patrimoniale tongienne. La critique des acteurs, quant à elle, repose sur des oppositions entre «bonne » et « mauvaise » pratique, les mises en scène touristiques appartenant à la seconde.

39 L'étude de ces discours sur les médiations touristiques du lakalaka révèle donc une certaine persistance des idées et valeurs associées depuis longtemps au patrimoine - 
l'importance de pérenniser dans une forme stable, l'idée qu'il y aurait des critères « objectifs » permettant de distinguer le « vrai » du « faux », la « copie » du « modèle ». Dans le cas traité ici, cette persistance est le fait des acteurs locaux, notamment des acteurs de la patrimonialisation et/ou des « experts » du lakalaka (punake). Je rejoins donc Frédéric Maguet (2011 : 70) lorsque ce dernier affirme que le discours de «l'authentique », qui insiste notamment sur la transmission et l'ancienneté des pratiques, peut être réintroduit par la bande dans le cadre des politiques du PCI, malgré des tentatives pour rompre avec celui-ci. Ainsi, la convention de 2003 n'inaugure sans doute pas de manière aussi radicale qu'on le prétend parfois une nouvelle « ère » patrimoniale.

Cette pesanteur des conceptions antérieures du patrimoine s'explique peut-être plus par les enjeux locaux liés à l'application de la convention pour la sauvegarde du PCI que par le manque d'innovation apportée par la convention elle-même. En effet, cette dernière semble présenter suffisamment de plasticité - en raison notamment de ses contradictions internes - pour pouvoir être adaptée ou appropriée en fonction des enjeux et des contextes locaux. Comme le dit Jean-Louis Tornatore, "prendre la convention, c'est la plier à sa tradition en s'y pliant, c'est s'y adapter en l'adaptant » (2012 : 7). Plutôt que de parler d'un " régime de patrimonialité » qui s'affirmerait de manière uniforme à l'échelle internationale, il semble préférable de parler de " régimes patrimoniaux " ( heritage regimes ») au pluriel, comme le proposent Regina Bendix, Adityah Eggert et Armika Peselmann (2012), ceux-ci résultant de l'articulation entre des politiques locales ou nationales de préservation de la culture et d'un régime patrimonial international, exprimé et défini par les conventions de l'Unesco notamment. Dans le cas de Tonga, c'est dans le contexte de la réforme politique et de l'augmentation des flux migratoires que les enjeux du processus de patrimonialisation doivent être compris. La perpétuation d'un « discours de l'authentique » fondé sur une opposition entre la « copie » et le « modèle » - ou des pratiques culturelles stables contre des pratiques culturelles dévoyées par la mondialisation - peut présenter certains avantages dans un contexte de changement politique rapide, parce qu'elle offre une forme de légitimation du système politique monarchique. On peut en effet faire l'hypothèse que le gouvernement tongien veut sans doute moins assurer la relation des Tongiens à leur histoire que leurs « bonnes relations à la bonne histoire » (Hertz \& Chappaz-Wirthner, $2012:$ 7).

41 Il est nécessaire de dépasser ce simple constat, qui somme toute ne fait qu'illustrer des mécanismes connus des processus de patrimonialisation, souvent décrits comme des constructions façonnées par des enjeux sociopolitiques actuels. Ce cas d'étude ouvre en effet des perspectives pour éclairer sous un jour nouveau les changements sociaux qui prennent place à Tonga. Si d'un côté, la patrimonialisation du lakalaka semble participer à la légitimation du régime politique en place, d'un autre côté, l'étude des dispositifs de médiation touristique du lakalaka révèle que lorsque ceux-ci sont pratiqués dans ces contextes, ils obéissent à des logiques sociales certes très différentes de celles pratiquées dans les contextes cérémoniels, mais ils n'en servent pas moins des processus de constructions identitaires, qui s'inscrivent dans un contexte social en mutation. Le discours du maître de cérémonie cité plus haut ne définit par exemple pas la tradition tongienne par l'importance de son système monarchique, auquel il est fait allusion au passé, mais plutôt en mettant l'accent sur la spécificité des relations hommes/femmes. La position sociale de ce maître de cérémonie, qui a vécu à l'étranger de nombreuses années et qui doit sa réussite sociale à ses activités économiques et non à son rang, peut sans doute expliquer en partie son point de vue. Les médiations touristiques du lakalaka entraînent donc un renouvellement des images et des valeurs associées à cette pratique. C'est en prenant en compte les différents contextes de pratique du lakalaka, et en examinant en même temps le processus de patrimonialisation et les occurrences de la pratique qui échappent à cette forme d'institutionnalisation, qu'il est possible de comprendre les mécanismes et les enjeux des changements sociaux et politiques à Tonga, et notamment la manière dont ceux-ci se traduisent dans des définitions multiples de l'identité tongienne. 


\section{Bibliographie}

Andrieu (Sarah). 2010. Performances et patrimonialisations du Wayang Golek sundanais (Java Ouest, Indonésie). Th. Doct : anthropologie, EHESS, Paris.

Bendix (Regina), Eggert (Aditya) \& Peselmann (Arnika) (sous la dir. de). 2012. The Heritage Regimes and the State. Göttingen : Göttingen University Press.

Besnier (Niko). 2011. On the Edge of the Global. Modern Anxieties in a Pacific Island Nation. Stanford: Stanford University Press.

Bortolotto (Chiara). 2007. «From Objects to Processes. Unesco's Intangible Cultural Heritage ». Journal of Museum Ethnography, 19, p. 21-33.

Bortolotto (Chiara) (sous la dir. de). 2011. le Patrimoine culturel immatériel. Enjeux d'une nouvelle catégorie. Paris : Éd. de la Maison des Sciences de l'Homme. (Ethnologie de la France.)

Ciarcia (Gaëtano). 2006. " La perte durable. Étude sur la notion de "patrimoine immatériel” ». les Carnets $d u$ lahic. Publication ligne <http://www.iiac.cnrs.fr/lahic/sites/lahic/IMG/pdf/Ciarcia_perte_durable.pdf>. Consulté le 2 mars 2013.

Condevaux (Aurélie). 2011. "Contextualisation of Dances in Tourism: A Tongan Case Study ». The Journal of the Polynesian Society, 120, p. 269-291.

Cousin (Saskia). 2008, "L'Unesco et la doctrine du tourisme culturel. Généalogie d'un "bon" tourisme ». Civilisations, 1-2, p. 41-37.

Douaire-Marsaudon (Françoise). 1998. les Premiers Fruits. Parenté, identité sexuelle etpouvoirs en Polynésie occidentale (Tonga, Wallis et Futuna). Paris : Éd. de la Maison des Sciences de l'Homme.

Gravari-Barbas (Maria). 2009. « Entre mise en tourisme et affirmation culturelle. La mise en valeur des patrimoines africains-américains », p. 75-102, in Culture, tourisme et développement, les voies d'un rapprochement / sous la direction de Claude Origet du Cluzeau \& Jean-Michel Tobelem. Paris : L'Harmattan.

Hafstein (Valdimar). 2009. " Intangible Heritage as a List. From Masterpieces to Representation », p. 93-108, in Intangible Heritage / sous la direction de Laurajane Smith \& Natsuko Akagawa. Londres : Routledge.

Hertz (Ellen) \& Chappaz-Wirthner (Suzanne). 2012. «Introduction. Le patrimoine a-t-il fait son temps ? ». Ethnographiques.org. Publication en ligne : <http://www.ethnographiques.org/2012/IMG/pdf/ArChappaz.pdf>. Consulté le 19 octobre 2012.

Johannson Fua (Seu'ula) et al. 2011. « Cultural Mapping, Planning and Policy: Tonga ». Secrétariat général de la Communauté du Pacifique pour le Ministry of Education, Women's Affairs and Culture, Nuku'alofa, Tonga. Publication en ligne : <http://www.spc.int/hdp/index2.php? option=com_docman\&task=doc_view\&gid=297\&Itemid=4>. Consulté le 19 octobre 2012.

Kaeppler (Adrienne). 1993. Poetry in Motion: Studies of Tongan Dance. Tongatapu: Vava'u Press.

Kirshenblatt-Gimblet (Barbara). 2006. «World Heritage and Cultural Economics », p. 161-202, in Museum Frictions. Public Cultures/Global Transformations / sous la direction d'lvan Karp et al. Durham et Londres : Duke University Press.

Maguet (Frédéric). 2011. " L'image des communautés dans l'espace public », p. 47-73, in le Patrimoine culturel immatériel. Enjeux d'une nouvelle catégorie / sous la direction de Chiara Bortolotto. Paris : Éd. de la Maison des Sciences de l’Homme. (Ethnologie de la France.)

Morisset (Lucie K.) \& Noppen (Luc). 2005. « Le patrimoine immatériel : Une arme à tranchants multiples ». Téoros. Publication en ligne : <http://teoros.revues.org/1500>. Consulté le 14 octobre 2012.

Munz (Hervé). 2012. "La fabrication et les usages politiques du "patrimoine horloger" dans le pays de Neuchâtel ». Ethnographiques.org. Publication en ligne : <http://www.ethnographiques.org/2012/Munz>. Consulté le 2 mars 2013.

Noyes (Dorothy). 2011. " La fête ou le fétiche, le geste ou la gestion. Du patrimoine culturel immatériel comme effet pervers de la démocratisation ", p. 123-148, in Le Patrimoine culturel immatériel. Enjeux d'une nouvelle catégorie / sous la direction de Chiara Bortolotto. Paris : Éd. de la Maison des Sciences de l'Homme. (Ethnologie de la France.)

Rautenberg (Michel). 2003. La Rupture patrimoniale. Bemin : Éd. À la croisée.

Smith (Laurajane) \& Akagawa (Natsuko) (sous la dir. de). 2009. Intangible heritage. Londres : Routledge.

Taumoefolau (Melenaite). 2004. "Queen Salote's Use of Heliaki », p. 103-145, in Songs and Poems of Queen Sâlote / sous la direction d'Elizabeth Wood-Ellem. Nuku'alofa : Vava'u Press. 
Tcherkézoff (Serge). 2003. "A Long and Unfortunate Voyage towards the "Invention" of the Melanesia/Polynesia Distinction 1595-1832 ». Journal of Pacific History, 38, p. 175-196.

Teilhet-Fisk (Jehanne H.). 1991. "To Beat or not to Beat, that is the Question: A Study on Acculturation and Change in Art-Making Process and its Relation to Gender Structures ». Pacific Studies, 14, p. 41-68.

Tornatore (Jean-Louis). 2012. « Retour d'anthropologie : "Le repas gastronomique des Français". Éléments d'ethnographie d'une distinction patrimoniale ». Ethnographiques.org. Publication en ligne : <http://www.ethnographiques.org/2012/Tornatore>. Consulté le 20 octobre 2012.

Turgeon (Laurier). 2010. «Introduction. Du matériel à l'immatériel. Nouveaux défis, nouveaux enjeux ». Ethnologie française, 40, p. 389-399.

DOI : 10.3917/ethn.103.0389

Unesco. 2003. "Convention pour la sauvegarde du patrimoine culturel immatériel ». Unesco. Publication en ligne : <http://www.unesco.org/culture/ichq/index.php?lg=en\&pg=00006>. Consulté le 23 octobre 2012.

Unesco. 2014. " Paris, dossiers CLT/CIH/ ITH/111 et CLT/CEH/ITH ». Archives Unesco. Publication en ligne : <http://www.unesco.org/archives/new2010/fr/index.html>. Consulté le 23 février 2014.

Wood-Ellem (Elizabeth). 2004. Songs and Poems of Queen Sâlote. Nuku'alofa : Vava'u Press.

\section{Notes}

2 Cette citation ainsi que les suivantes sont traduites par l'auteur.

3 Localement appelés "spectacles de fête et de danse tongienne ", " tongan feasts and dance shows » ou "floorshows ». Ce terme, qui désignait à l'origine des performances dansées dans des bars ou boîtes de nuit, tend aujourd'hui à être utilisé pour des spectacles de type cabaret. Il a acquis, dans le contexte tongien, un sens très spécifique : il désigne uniquement des spectacles de danses tongiennes et/ou « polynésiennes » exécutés pour des divertissements " populaires », non destinés au roi ou à sa famille par exemple. Le concept de « performance », que je préfère à celui de « spectacle » pour traduire ces expressions, permet de rendre compte de la nature particulière de l'interaction en jeu. Je propose de définir la performance comme un événement distingué du quotidien par divers moyens et motivé par la volonté de communiquer « quelque chose » à un public ou d'influencer celui-ci d'une manière ou d'une autre. Cette communication fait par ailleurs appel à de multiples registres sensoriels et utilise aussi bien le langage verbal, la manipulation de symboles ou d'objets, que la gestuelle corporelle. Le fait que cette définition mette en exergue la complexité de l'acte de communication à l'œuvre rend en soi le concept de performance plus adéquat que celui de spectacle pour désigner ces représentations touristiques.

\section{Pour citer cet article}

Référence papier Aurélie Condevaux, « Le patrimoine culturel immatériel à Tonga et ses médiations touristiques », Culture \& Musées, 23 | 2014, 43-65.

Référence électronique

Aurélie Condevaux, « Le patrimoine culturel immatériel à Tonga et ses médiations touristiques », Culture \& Musées [En ligne], 23 | 2014, mis en ligne le 19 juin 2018, consulté le 25 juin 2021. URL : http://journals.openedition.org/culturemusees/1344 ; DOI :

https://doi.org/10.4000/culturemusees.1344

\section{Cet article est cité par}

- Condevaux, Aurélie. (2015) La construction des patrimoines en question(s). DOI: 10.4000/books.psorbonne.7291

\section{Auteur}

\section{Aurélie Condevaux}


Suite à une thèse d'anthropologie soutenue en 2011, qui portait sur des « performances touristiques » en Nouvelle-Zélande et à Tonga, Aurélie Condevaux a mené des recherches postdoctorales sur la patrimonialisation du lakalaka - forme de discours poétique dansé et chanté - au sein du labex Création, Arts et Patrimoines, en association avec le musée du quai Branly. Elle est actuellement ATER à l'université de Poitiers et membre de Migrinter (UMR 7301). aurelie.condevaux-a[at]hotmail.fr

\section{Droits d'auteur}

Culture \& Musées 